Report no. $98 / 15$

\title{
Stiff Oscillatory Systems, Delta Jumps and White Noise
}

\author{
B. Cano ${ }^{1}$, A.M. Stuart ${ }^{2}$, E. Süli ${ }^{3}$ and J.O. Warren ${ }^{4}$
}

\begin{abstract}
Two model problems for stiff oscillatory systems are introduced. Both comprise a linear superposition of $N \gg 1$ harmonic oscillators used as a forcing term for a scalar ODE. In the first case the initial conditions are chosen so that the forcing term approximates a delta function as $N \rightarrow \infty$ and in the second case so that it approximates white noise. In both cases the fastest natural frequency of the oscillators is $\mathcal{O}(N)$.

The model problems are integrated numerically in the stiff regime where the time-step $\Delta t$ satisfies $N \Delta t=\mathcal{O}(1)$. The convergence of the algorithms is studied in this case in the limit $N \rightarrow \infty$ and $\Delta t \rightarrow 0$. For the white noise problem both strong and weak convergence are considered.
\end{abstract}

Subject classifications: AMS(MOS): 34A65, 60H10, 65L70, 82C80

Key words and phrases: stiff systems, stochastic differential equations, strong convergence, weak convergence

Oxford University Computing Laboratory

Numerical Analysis Group

Wolfson Building

Parks Road

Oxford OX1 3QD, England

December, 1998

\footnotetext{
${ }^{1}$ Departamento de Matemática Applicada y Computación, Facultad de Ciencias, Universidad de Valladolid, Valladolid, Spain. Supported by JCL VA36/98 and DGICYT PB95-705.

${ }^{2}$ Scientific Computing and Computational Mathematics Program, Durand 257, Stanford University, Stanford CA 94305-4040, USA. Supported by the National Science Foundation under grant DMS-95-04879 and by the EPSRC under grant GR/L82922.

${ }^{3}$ Oxford University Computing Laboratory, Wolfson Building, Parks Road, Oxford OX1 3QD.

${ }^{4}$ Scientific Computing and Computational Mathematics Program, Durand 266, Stanford University, Stanford CA 94305-4040, USA. Supported by the Department of Defense National Defense Science and Engineering Graduate Fellowship Program.
} 


\section{Introduction}

In the field of computational statistical mechanics, stiff oscillatory systems, with broad frequency spectra, often arise. It is hence of interest to develop a theory of the numerical analysis for such problems. In the area of stiff dissipative systems the understanding of numerical algorithms has been greatly enhanced by the study of a variety of simple model problems [2]; here we introduce, and then study numerical methods for, several model problems in stiff oscillatory systems. A review of existing literature in this area may be found in [7] and in section 6 of [8].

Section 2 introduces two basic models, both motivated by a simple mechanical description of a heat bath. In the first, a family of harmonic oscillators is used to construct an approximate delta function, through Fourier analysis, and this is used as a forcing term for a scalar ODE. In the second, a family of harmonic oscillators is used to construct an approximation to white noise, again through Fourier analysis, and this is used as a forcing term for a scalar ODE. For both models the fastest natural frequency of the oscillators is $\mathcal{O}(N)$, where $N$ is the number of oscillators; the approximation to a delta function or to white noise becomes exact as $N \rightarrow \infty$; theorems making this precise are given.

Our aim is to study the convergence of numerical methods for these models in the regime

$$
N \Delta t \text { fixed; } \quad \Delta t \rightarrow 0, N \rightarrow \infty \text {. }
$$

In statistical mechanics, the interaction of an observable with a heat bath can be modelled by a purely mechanical system with random data $[3,9,10]$. That work motivates the choice of constructing a delta function and white noise through families of oscillators since there Fourier-based approximations of delta-function induced jumps arise in the modelling of the energyloss mechanism from the observable to the heat bath, whilst the Fourierbased white noise models the (expected) energy gain mechanism, given a random distribution on the initial data. Numerical experiments in [8] study the observable/heat-bath interaction numerically under the limit (1.1). The purpose of this paper is to further understand the numerical analysis of such problems by isolating, and then studying separately, the approximation of delta functions and white noise through numerically approximated Fourier series.

Section 3 contains an analysis of the delta jump model and convergence is studied in a discrete $L^{2}$ norm under the limit process (1.1). In section 4 the white noise problem is studied in a discrete $L^{\infty}$ norm in time, from the point of view of strong convergence (with respect to an appropriate probability measure) under (1.1). In section 5 the white noise problem is again studied, but from the viewpoint of weak convergence under (1.1).

Our results show that:

- For the delta jump model only certain special methods exhibit convergence to the correct limit under (1.1). Other methods accurately 
approximate an incorrect limit.

- For the delta jump model convergence under (1.1) is at a reduced rate when compared with the fixed $N, \Delta t \rightarrow 0$ behaviour.

- For the white noise model similar reduced rates of convergence are observed.

- For the white noise model the rates of convergence are better for weak than for strong convergence, a situation familiar from standard approximation theory for SDEs [5].

\section{Model Problems}

Consider the equations

$$
\begin{gathered}
\ddot{u}_{j}+j^{2} u_{j}=0, \\
u_{j}(0)=a_{j}, \quad \dot{u}_{j}(0)=0, \quad j=0, \ldots, N,
\end{gathered}
$$

and

$$
\begin{gathered}
\dot{z}_{\mathrm{N}}=f\left(z_{\mathrm{N}}\right)+H_{\mathrm{N}}(t), \\
z_{\mathrm{N}}(0)=z_{0},
\end{gathered}
$$

where

$$
H_{\mathrm{N}}(t):=\sum_{j=0}^{N} u_{j}(t)
$$

We consider two choices for the $\left\{a_{j}\right\}_{j=0}^{N}$ : the first is

$$
a_{0}=\frac{1}{2}, \quad a_{j}=1, j \geq 1
$$

The second is

$$
a_{0}=\frac{1}{\sqrt{\pi}} \eta_{0}, \quad a_{j}=\sqrt{\frac{2}{\pi}} \eta_{j}, j \geq 1 ;
$$

here the $\eta_{j}$ are IID Gaussian random variables with mean 0 and variance 1 .

Throughout the following we assume that $f \in C^{\infty}(\mathbb{R}, \mathbb{R})$ and satisfies the global Lipschitz condition

$$
|f(x)-f(y)| \leq L|x-y| \quad \forall x, y \in \mathbb{R} .
$$

Formal calculations indicate that for [MP1], $0 \leq t \leq \pi$ and $N$ large, $z_{\mathrm{N}}$ should behave like $z$ solving

$$
\dot{z}=f(z), \quad z(0)=z_{0}+\frac{\pi}{2} .
$$


For [MP2] the analogous formal limit is the SDE

$$
d z=f(z) d t+d W, \quad z(0)=z_{0},
$$

where $W$ is a standard Brownian motion on $0 \leq t \leq \pi$. The following three results make this intuition precise.

Theorem 2.1 Consider $z_{N}(t)$ solving [MP1] and $z(t)$ solving (2.5). Then, for $T \in(0, \pi]$,

$$
\left\|z(\cdot)-z_{N}(\cdot)\right\|_{L^{2}(0, T)}^{2} \leq \frac{C(T)}{N} .
$$

Theorem 2.2 Consider $z_{N}(t)$ solving [MP2] and $z(t)$ solving (2.6). Then, for $T \in(0, \pi]$,

$$
\sup _{t \in(0, T)} \mathbb{E}\left|z(t)-z_{N}(t)\right|^{2} \leq \frac{C(T)}{N}
$$

It is often the case that weak convergence results can be obtained at faster rates than strong convergence and we now demonstrate this. We consider expectations of functions $g: \mathbb{R} \rightarrow \mathbb{R}$ whose Fourier transform $\hat{g}$ satisfies

Hypothesis $\mathbf{H}$ There exists a real number $\beta>1$ and a positive constant $C_{1}$ such that

$$
|\hat{g}(k)| \leq C_{1}(1+|k|)^{-\beta} \quad \forall k \in \mathbb{R}
$$

In the following theorem, in Proposition 2.5 and in section 5, we consider the case $f \equiv 0$. Thus $z$ solving (2.6) is a pure Brownian motion. This allows relatively straightforward analysis using Fourier techniques; more sophisticated methods would be required to analyze the case of non-zero $f$.

Theorem 2.3 Let $f(z) \equiv 0$ and let $g: \mathbb{R} \rightarrow \mathbb{R}$ satisfy Hypothesis H. Consider $z_{N}(t)$ solving [MP2] and $z(t)$ solving $(2.6)$. Then, for $T \in(0, \pi]$,

$$
\sup _{z_{0} \in R}\left|\mathbb{E} g(z(T))-\mathbb{E} g\left(z_{N}(T)\right)\right| \leq \begin{cases}C N^{(1-\beta) / 2}, & 1<\beta<3, \\ C N^{-1} \log (1+N), & \beta=3, \\ C N^{-1}, & \beta>3,\end{cases}
$$

where $C=C\left(\beta, C_{1}\right)$ with $\beta$ and $C_{1}$ as in Hypothesis $H$.

These theorems are proved at the end of the section. In the course of proving Theorem 2.3 we also estimate the difference between $p(z, t)$ the probability density function (pdf) for $z(t)$ solving (2.6), and $\bar{p}_{\mathrm{N}}(z, t)$ the pdf for $z_{\mathrm{N}}(t)$ solving [MP2]; a proposition summarizing these estimates may be found after the proof of Theorem 2.3.

In sections 3, 4 and 5 we consider numerical solutions of (2.1), (2.2) in the regime (1.1) and address the question of whether the numerical approximations identify the correct limiting behaviour, as given in Theorems 2.1, 
2.2 and 2.3. We solve (2.1) by a family of parameterized energy conserving methods, ${ }^{1}$ namely

$$
\begin{gathered}
U_{j}^{n+1}-2 U_{j}^{n}+U_{j}^{n-1}+j^{2} \Delta t^{2} U_{j}^{n}=0 \\
U_{j}^{0}=a_{j}, \quad U_{j}^{1}=a_{j}\left[1-\alpha j^{2} \Delta t^{2}\right]
\end{gathered}
$$

and (2.2) by the $\theta$-method, for $t^{n}=n \Delta t$,

$$
\begin{aligned}
Z^{n+1}-Z^{n}= & \Delta t\left[\theta f\left(Z^{n+1}\right)+(1-\theta) f\left(Z^{n}\right)\right] \\
& +\Delta t\left[\theta H_{\mathrm{N}}^{\Delta t}\left(t^{n+1}\right)+(1-\theta) H_{\mathrm{N}}^{\Delta t}\left(t^{n}\right)\right]
\end{aligned}
$$

with $Z^{0}=z_{0}$ and where $H_{\mathrm{N}}^{\Delta t}\left(t^{n}\right)$ is the approximation to $H_{\mathrm{N}}\left(t^{n}\right)$ with the $u_{j}\left(t^{n}\right)$ computed through (2.9).

The following lemma, taken from [8], will be useful in the study of (2.9), $(2.10)$.

Lemma 2.4 The sequence $\left\{Z^{n}\right\}_{n \geq 0}$ generated by (2.10) satisfies

$$
\begin{aligned}
Z^{n}= & z_{0}+\Delta t \sum_{m=0}^{\prime n} f\left(Z^{m}\right)+(n \Delta t) a_{0}+\sum_{j=1}^{N} a_{j} \gamma_{j} \sin \left(\phi_{j} n\right) \\
& +(1-\theta-\alpha) \Delta t \sum_{j=1}^{N} 2 a_{j} \sin ^{2}\left(\phi_{j} n / 2\right),
\end{aligned}
$$

where $\sum_{m=0}^{\prime n}$ denotes a sum with weight $(1-\theta)$ on $m=0, \theta$ on $m=n$, and 1 otherwise. Furthermore $\phi_{j}$ and $\gamma_{j}$ are given by

$$
\cos \phi_{j}=1-\frac{1}{2} j^{2} \Delta t^{2}
$$

and

$$
\gamma_{j}=\frac{\sqrt{1-\frac{1}{4} j^{2} \Delta t^{2}}}{j}+\frac{\left(\alpha-\frac{1}{2}\right)\left(\frac{1}{2}-\theta\right) j \Delta t^{2}}{\sqrt{1-\frac{1}{4} j^{2} \Delta t^{2}}}
$$

We now prove Theorems $2.1-2.3$.

Proof of Theorem 2.1 In this case

$$
H_{\mathrm{N}}(t)=\frac{d h_{\mathrm{N}}}{d t}(t)
$$

where

$$
h_{\mathrm{N}}(t)=\frac{t}{2}+\sum_{j=1}^{N} \frac{\sin (j t)}{j} .
$$

Straightforward Fourier analysis shows that

$$
\left\|\left(h_{\mathrm{N}}(\cdot)-\frac{\dot{2}}{2}\right)-\left(\frac{\pi-\cdot}{2}\right)\right\|_{L^{2}(0, \pi)}^{2} \leq \frac{C}{N} .
$$

\footnotetext{
${ }^{1}$ For $j \Delta t \in[0,2]$ the method (2.9) conserves a small perturbation of the energy of the underlying harmonic oscillator.
} 
Writing (2.2), (2.5) as integral equations gives

$$
\begin{gathered}
z_{\mathrm{N}}(t)=z_{0}+\int_{0}^{t} f\left(z_{\mathrm{N}}(s)\right) d s+h_{\mathrm{N}}(t), \\
z(t)=z_{0}+\int_{0}^{t} f(z(s)) d s+\frac{\pi}{2} .
\end{gathered}
$$

Subtracting and defining $e_{\mathrm{N}}(t)=z_{\mathrm{N}}(t)-z(t)$ gives

$$
e_{\mathrm{N}}(t)=\int_{0}^{t}\left[f\left(z_{\mathrm{N}}(s)\right)-f(z(s))\right] d s+\left[h_{\mathrm{N}}(t)-\frac{\pi}{2}\right] .
$$

Taking $L^{2}$-norms and using (2.11) gives, for $t \in[0, \pi]$,

$$
\left\|e_{\mathrm{N}}(\cdot)\right\|_{L^{2}(0, t)}^{2} \leq \frac{2 C}{N}+2 \pi L^{2} \int_{0}^{t}\left\|e_{\mathrm{N}}(\cdot)\right\|_{L^{2}(0, s)}^{2} d s .
$$

A Gronwall argument gives the desired result.

Proof of Theorem 2.2 In the case of [MP2] it follows that

$$
h_{\mathrm{N}}(t)=\frac{\eta_{0} t}{\sqrt{\pi}}+\sum_{j=1}^{N} \sqrt{\frac{2}{\pi}} \eta_{j} \frac{\sin (j t)}{j} .
$$

From [6] (Chapter 2, Theorem 2.5) it is known that, with probability one,

$$
h_{\mathrm{N}}(t) \rightarrow W(t)
$$

uniformly for $t \in[0, \pi]$, where $W(t)$ is standard Brownian motion. Hence, almost surely,

$$
W(t)=\frac{\eta_{0} t}{\sqrt{\pi}}+\sum_{j=1}^{\infty} \sqrt{\frac{2}{\pi}} \eta_{j} \frac{\sin (j t)}{j}
$$

from which it follows that, for each $t \in[0, \pi]$,

$$
\begin{aligned}
\mathbb{E}\left|h_{\mathrm{N}}(t)-W(t)\right|^{2} & =\mathbb{E} \sum_{j, k \geq N+1} \frac{2}{\pi} \eta_{j} \eta_{k} \frac{\sin (j t) \sin (k t)}{j k} \\
& =\sum_{j \geq N+1} \frac{2}{\pi} \frac{\sin ^{2}(j t)}{j^{2}} \\
& \leq \frac{2 C}{\pi N} .
\end{aligned}
$$

Rewriting (2.6) as an integral equation gives

$$
z(t)=z_{0}+\int_{0}^{t} f(z(s)) d s+W(t) .
$$

Subtracting from equation $(2.12)$ and defining $e_{\mathrm{N}}(t)=z_{\mathrm{N}}(t)-z(t)$ gives

$$
e_{\mathrm{N}}(t)=\int_{0}^{t}\left[f\left(z_{\mathrm{N}}(s)\right)-f(z(s))\right] d s+\left[h_{\mathrm{N}}(t)-W(t)\right] .
$$


Thus

$$
\begin{aligned}
\mathbb{E}\left|e_{\mathrm{N}}(t)\right|^{2} & \leq 2 \mathbb{E}\left\{\int_{0}^{t}\left[f\left(z_{\mathrm{N}}(s)\right)-f(z(s))\right] d s\right\}^{2}+\frac{4 C}{\pi N} \\
& \leq 2 \pi L^{2} \int_{0}^{t} \mathbb{E}\left|e_{\mathrm{N}}(s)\right|^{2} d s+\frac{4 C}{\pi N} .
\end{aligned}
$$

A Gronwall argument gives the desired result.

Proof of Theorem 2.3 We wish to study the rate of convergence of the quantity

$$
\left|\mathbb{E} g(z(t))-\mathbb{E} g\left(z_{N}(t)\right)\right|
$$

to 0 as $N \rightarrow \infty$ in the case when

$$
f(\cdot) \equiv 0 .
$$

The pdf $p(z, t)$ for the problem (2.6) under (2.18) is the solution of the parabolic initial-value problem

$$
\left\{\begin{aligned}
\frac{\partial p}{\partial t} & =\frac{1}{2} \frac{\partial^{2} p}{\partial z^{2}}, \\
p(z, 0) & =\delta\left(z-z_{0}\right) .
\end{aligned}\right.
$$

Similarly, for each fixed $\omega:=\left\{\eta_{j}\right\}_{j \geq 0}$, the pdf $p_{\mathrm{N}}(z, t ; \omega)$ for the problem (2.2) satisfies the hyperbolic initial value problem

$$
\left\{\begin{array}{c}
\frac{\partial p_{\mathrm{N}}}{\partial t}+\frac{\partial}{\partial z}\left(\frac{d h_{\mathrm{N}}}{d t} p_{\mathrm{N}}\right)=0 \\
p_{\mathrm{N}}(z, 0 ; \omega)=\delta\left(z-z_{0}\right)
\end{array}\right.
$$

where $h_{\mathrm{N}}(t)=h_{\mathrm{N}}(t ; \omega)$ is given by $(2.14)$. We set

$$
\bar{p}_{N}(z, t)=\mathbb{E} p_{\mathrm{N}}(z, t ; \omega),
$$

and note that

$$
\begin{aligned}
\mathbb{E} g(z(t)) & =\int_{-\infty}^{\infty} p(z, t) g(z) d z, \\
\mathbb{E} g\left(z_{N}(t)\right) & =\int_{-\infty}^{\infty} \bar{p}_{N}(z, t) g(z) d z .
\end{aligned}
$$

Thus we shall first estimate the closeness of $\bar{p}_{N}$ to $p$, and thereby derive bounds on the quantity (2.17).

We define

$$
\hat{p}_{\mathrm{N}}(k, t ; \omega)=\int_{-\infty}^{\infty} \mathrm{e}^{i k z} p_{\mathrm{N}}(z, t ; \omega) d z
$$

Applying the Fourier transform to (2.20), it is a straightforward matter to check that

$$
\frac{\partial \hat{p}_{N}}{\partial t}-i k \frac{d h_{\mathrm{N}}}{d t} \hat{p}_{\mathrm{N}}=0, \quad \hat{p}_{\mathrm{N}}(k, 0 ; \omega)=\mathrm{e}^{i k z_{0}}
$$


and therefore

$$
\hat{p}_{\mathrm{N}}(k, t ; \omega)=\mathrm{e}^{i k z_{0}} \mathrm{e}^{i k h_{\mathrm{N}}(t ; \omega)},
$$

where we have made use of the fact that $h_{\mathrm{N}}(0 ; \omega)=0$. Hence,

$$
p_{\mathrm{N}}(z, t ; \omega)=\frac{1}{2 \pi} \int_{-\infty}^{\infty} \mathrm{e}^{i k\left(z_{0}-z\right)} e^{i k h_{\mathrm{N}}(t ; \omega)} d k
$$

so that

$$
\bar{p}_{\mathrm{N}}(z, t)=\frac{1}{2 \pi} \int_{-\infty}^{\infty} \mathrm{e}^{i k\left(z_{0}-z\right)} \psi_{N}(k, t) d k
$$

where $\psi_{N}(k, t)$ is the characteristic function for $h_{\mathrm{N}}(t ; \omega)$; i.e.

$$
\begin{aligned}
\psi_{N}(k, t) & =\mathbb{E} \exp \left\{i k h_{\mathrm{N}}(t ; \omega)\right\} \\
& =\exp \left\{-\frac{k^{2} t^{2}}{2 \pi}-\frac{k^{2}}{\pi} \sum_{j=1}^{N} \frac{\sin ^{2} j t}{j^{2}}\right\} \\
& =\exp \left\{-\frac{k^{2}}{2}\left[\frac{t^{2}}{\pi}+\frac{2}{\pi} \sum_{j=1}^{N} \frac{\sin ^{2} j t}{j^{2}}\right]\right\} \\
& =\exp \left\{-\frac{k^{2}}{2}\left[\frac{t^{2}}{\pi}+\frac{2}{\pi} \sum_{j=1}^{N} \frac{1-\cos (2 j t)}{2 j^{2}}\right]\right\} \\
& =\exp \left\{-\frac{k^{2}}{2}\left[\frac{t^{2}}{\pi}+\frac{1}{\pi} \sum_{j=1}^{N} \frac{1}{j^{2}}-\frac{1}{\pi} \sum_{j=1}^{N} \frac{\cos (2 j t)}{j^{2}}\right]\right\} .
\end{aligned}
$$

As

$$
\sum_{j=1}^{\infty} \frac{1}{j^{2}}=\frac{\pi^{2}}{6}
$$

and, by straightforward Fourier series expansion,

$$
\frac{t^{2}}{\pi}-t=-\frac{\pi}{6}+\frac{1}{\pi} \sum_{j=1}^{\infty} \frac{\cos (2 j t)}{j^{2}}
$$

we deduce (formally, at least) that, as $N \rightarrow \infty$,

$$
\begin{aligned}
& \psi_{N}(k, t) \rightarrow \exp \left\{-\frac{1}{2} k^{2} t\right\}, \\
& \bar{p}_{\mathrm{N}}(z, t) \rightarrow \frac{1}{2 \pi} \int_{-\infty}^{\infty} \mathrm{e}^{i k\left(z-z_{0}\right)} \mathrm{e}^{-\frac{1}{2} k^{2} t} d k=p(z, t) .
\end{aligned}
$$

We now aim to make these statements precise. 
Since the Fourier series on the right-hand side of (2.25) converges uniformly for $t \in[0, \pi]$ to the function on the left-hand side of (2.25),

$$
\mathrm{e}^{-\frac{1}{2} k^{2} t}=\exp \left\{-\frac{1}{2} k^{2}\left[\frac{t^{2}}{\pi}+\frac{\pi}{6}-\frac{1}{\pi} \sum_{j=1}^{\infty} \frac{\cos (2 j t)}{j^{2}}\right]\right\} \quad \forall t \in[0, \pi] .
$$

Also, from (2.24) we have that

$$
\psi_{N}(k, t)=\exp \left\{-\frac{1}{2} k^{2}\left[\frac{t^{2}}{\pi}+\frac{1}{\pi} \sum_{j=1}^{N} \frac{1}{j^{2}}-\frac{1}{\pi} \sum_{j=1}^{N} \frac{\cos (2 j t)}{j^{2}}\right]\right\} .
$$

Alternatively, the last two lines can be rewritten as, respectively,

$$
\begin{aligned}
\mathrm{e}^{-\frac{1}{2} k^{2} t} & =\exp \left\{-\frac{1}{2} k^{2}\left[\frac{t^{2}}{\pi}+S_{1}^{\infty}\right]\right\}, \\
\psi_{N}(k, t) & =\exp \left\{-\frac{1}{2} k^{2}\left[\frac{t^{2}}{\pi}+S_{1}^{N}\right]\right\},
\end{aligned}
$$

where

$$
S_{1}^{\infty}=\frac{2}{\pi} \sum_{j=1}^{\infty} \frac{\sin ^{2} j t}{j^{2}}, \quad S_{1}^{N}=\frac{2}{\pi} \sum_{j=1}^{N} \frac{\sin ^{2} j t}{j^{2}}
$$

On subtracting (2.27) from (2.26) and noting that

$$
\left|\mathrm{e}^{-a}-\mathrm{e}^{-b}\right| \leq\left[1-\mathrm{e}^{-|a-b|}\right], \quad a, b \geq 0
$$

we deduce that

$$
\left|e^{-\frac{1}{2} k^{2} t}-\psi_{N}(k, t)\right| \leq \mathrm{e}^{-\frac{k^{2} t^{2}}{2 \pi}}\left[1-\exp \left(-\frac{k^{2}}{\pi} \sum_{j \geq N+1} \frac{\sin ^{2} j t}{j^{2}}\right)\right]
$$

Further, since

$$
\sum_{j \geq N+1} \frac{\sin ^{2} j t}{j^{2}} \leq \sum_{j \geq N+1} \frac{1}{j^{2}} \leq \frac{1}{N}
$$

it follows from (2.30) that

$$
\left|\mathrm{e}^{-\frac{1}{2} k^{2} t}-\psi_{N}(k, t)\right| \leq \mathrm{e}^{-\frac{k^{2} t^{2}}{2 \pi}}\left[1-\mathrm{e}^{-\frac{k^{2}}{\pi N}}\right] .
$$

Now we consider (2.21) and (2.22). By virtue of Parseval's identity

$$
\begin{aligned}
\mathbb{E} g(z(t))-\mathbb{E} g\left(z_{N}(t)\right) & =\int_{-\infty}^{\infty}\left[p(z, t)-\bar{p}_{N}(z, t)\right] g(z) d z \\
& =\frac{1}{2 \pi} \int_{-\infty}^{\infty} \mathrm{e}^{i k\left(z_{0}-z\right)}\left[e^{-\frac{1}{2} k^{2} t}-\psi_{N}(k, t)\right] \hat{g}(k) d k
\end{aligned}
$$


Thus,

$$
\left|\mathbb{E} g(z(t))-\mathbb{E} g\left(z_{N}(t)\right)\right| \leq \frac{1}{2 \pi} \int_{-\infty}^{\infty}\left|\mathrm{e}^{-\frac{1}{2} k^{2} t}-\psi_{N}(k, t)\right||\hat{g}(k)| d k .
$$

Applying Hypothesis H in (2.32) and recalling (2.31), it follows that

$$
\left|\mathbb{E} g(z(t))-\mathbb{E} g\left(z_{N}(t)\right)\right| \leq \frac{C_{1}}{\pi} \int_{0}^{\infty} \mathrm{e}^{-\frac{k^{2} t^{2}}{2 \pi}}\left[1-\mathrm{e}^{-\frac{k^{2}}{\pi N}}\right](1+k)^{-\beta} d k .
$$

In order to complete the analysis, it remains to bound the right-hand side in (2.33). First, we note that

$$
1-\mathrm{e}^{-a} \leq \min (1, a), \quad a \geq 0 .
$$

Applying this in (2.33) with $a=k^{2} /(\pi N)$, it follows that

$$
\left|\mathbb{E} g(z(t))-\mathbb{E} g\left(z_{N}(t)\right)\right| \leq \frac{C_{1}}{\pi} \int_{0}^{\infty} \mathrm{e}^{-\frac{k^{2} t^{2}}{2 \pi}}(1+k)^{-\beta} \min \left(1, \frac{k^{2}}{\pi N}\right) d k .
$$

In Appendix A we show that

$$
\int_{0}^{\infty} \mathrm{e}^{-\frac{k^{2} t^{2}}{2 \pi}}(1+k)^{-\beta} \min \left(1, \frac{k^{2}}{\pi N}\right) d k \leq \begin{cases}C N^{(1-\beta) / 2}, & 1<\beta<3 \\ C N^{-1} \log (1+N), & \beta=3 \\ C N^{-1}, & \beta>3\end{cases}
$$

where $C=C(\beta)$ is a positive constant. Finally, inserting (2.36) into (2.35), we arrive at the required bound.

By using the estimates from the proof of Theorem 2.3, some simple calculations given in Appendix B enable the proof of the following:

Proposition 2.5 Let $f(z) \equiv 0$. Consider $\bar{p}_{N}(z, t)$, the pdf for $z_{N}(t)$ solving [MP2] and $p(z, t)$ the pdf for $z(t)$ solving (2.6). Then, for $T \in[0, \pi]$,

$$
\int_{0}^{T} t^{\alpha}\left\|\bar{p}_{N}(\cdot, t)-p(\cdot, t)\right\|_{L_{\infty}(\mathbb{R})} d t \leq C \begin{cases}N^{-\frac{\alpha}{2}} & \text { for } 0<\alpha<2 \\ N^{-1} \log (1+N) & \text { for } \alpha=2 \\ N^{-1} & \text { for } \alpha>2\end{cases}
$$

where $C=C(\alpha)$ is a positive constant. Furthermore,

$$
\int_{0}^{T} t^{\alpha}\left\|\bar{p}_{N}(\cdot, t)-p(\cdot, t)\right\|_{L^{2}(\mathbb{R})} d t \leq C \begin{cases}N^{-\left(\frac{\alpha}{2}+\frac{1}{4}\right)} & \text { for }-\frac{1}{2}<\alpha<\frac{3}{2} \\ N^{-1} \log (1+N) & \text { for } \alpha=3 / 2 \\ N^{-1} & \text { for } \alpha>\frac{3}{2}\end{cases}
$$

where, again, $C=C(\alpha)$ is a positive constant. 


\section{Numerical Approximation of Jumps}

To state our basic result it will be useful to introduce some notation. Given a vector $\left(w_{1}, \ldots, w_{m-1}\right)^{T}$ we define

$$
w=\left(w_{1}, \ldots, w_{m-1}\right)^{T}
$$

this notation will be extended to vectors other than $w$. We also define

$$
s^{(j)}=(0, \sin (j \Delta t), \sin (j 2 \Delta t), \ldots, \sin (j[m-1] \Delta t))^{T}
$$

and

$$
\bar{s}^{(j)}=\left(0, \sin \left(\phi_{j}\right), \sin \left(2 \phi_{j}\right), \ldots, \sin \left([m-1] \phi_{j}\right)\right)^{T} .
$$

Then

$$
\begin{gathered}
\langle v, w\rangle_{m}=\Delta t \sum_{n=0}^{m-1} v_{n} w_{n}, \\
\|w\|_{L^{2}(0, m \Delta t)}^{2}=\langle w, w\rangle_{m} .
\end{gathered}
$$

For purposes of comparison with the numerical method, it will be useful to project the solution of (2.5) onto the grid by defining $z^{n}=z(n \Delta t)$.

Theorem 3.1 Consider $\left\{Z^{n}\right\}_{n \geq 0}$ solving [MP1] numerically with $\theta+\alpha=1$ and $N \Delta t=r<2$ and $\left\{z^{n}\right\}_{n \geq 0}$ the projection of $z(t)$ solving (2.5) onto the grid. Then, for $n \Delta t \in[0, \pi]$,

$$
\|z-Z\|_{L^{2}(0, n \Delta t)}^{2} \leq C(n \Delta t) \log \left|\Delta t^{-1}\right| \Delta t^{2 / 3} .
$$

Proof For simplicity we assume that there is an integer $M$ such that $M \Delta t=\pi$. Other choices of $\Delta t$ can be handled by approximation. By (2.11) we may write (2.13) as

$$
z(t)=z_{0}+\int_{0}^{t} f(z(s)) d s+\frac{t}{2}+\sum_{j=1}^{\infty} \frac{\sin (j t)}{j} .
$$

By Lemma 2.4 we obtain

$$
\begin{aligned}
z^{n}-Z^{n}= & \int_{0}^{t} f(z(s)) d s-\Delta t \sum_{m=0}^{\prime n} f\left(Z^{m}\right) \\
& +\sum_{j \geq N+1} a_{j} \frac{\sin (j n \Delta t)}{j}+\sum_{j=1}^{N} a_{j}\left(j^{-1}-\gamma_{j}\right) \sin (j n \Delta t) \\
& +\sum_{j=1}^{N} a_{j} \gamma_{j}\left[\sin (j n \Delta t)-\sin \left(\phi_{j} n\right)\right] \\
& +(\theta+\alpha-1) \Delta t \sum_{j=1}^{N} 2 a_{j} \sin ^{2}\left(\phi_{j} n / 2\right),
\end{aligned}
$$

where for $[\mathrm{MP} 1] a_{j}=1, j \geq 1$. Henceforth in this proof we set $\theta+\alpha=1$. Now the regularity of solutions to ODEs implies that

$$
\sup _{t \in[0, \pi]}\left|\int_{0}^{t} f(z(s)) d s-\Delta t \sum_{m=0}^{n} f\left(z^{m}\right)\right| \leq C \Delta t .
$$


Thus, using the fact that $N \Delta t=r$ and $n \Delta t \in[0, \pi]$, we obtain for $e^{n}=$ $z^{n}-Z^{n}$,

$$
\begin{aligned}
\|e\|_{L^{2}(0, n \Delta t)}^{2} \leq & 5 \pi L^{2}\left[\sum_{m=0}^{\prime n}\|e\|_{L^{2}(0, m \Delta t)}^{2}\right] \\
& +5 C^{2} \Delta t^{2}+\frac{5 \pi}{2} \sum_{j \geq N+1} \frac{1}{j^{2}}+\frac{5 \pi}{2} \sum_{j=1}^{N}\left(j^{-1}-\gamma_{j}\right)^{2} \\
& +5\left\|\sum_{j=1}^{N} \gamma_{j}\left[s^{(j)}-\bar{s}^{(j)}\right]\right\|_{L^{2}(0, M \Delta t)}^{2}
\end{aligned}
$$

By using

$$
1-(1-x)^{\frac{1}{2}} \leq x \quad \forall x \in[0,1]
$$

we obtain

$$
\left|\gamma_{j}-\frac{1}{j}\right|=\mathcal{O}\left(\Delta t^{2} j\right), \quad\left|\gamma_{j}\right|=\mathcal{O}\left(j^{-1}\right),
$$

so that

$$
2 \sum_{j=1}^{N}\left(j^{-1}-\gamma_{j}\right)^{2}=\mathcal{O}(\Delta t)
$$

Hence

$$
\begin{aligned}
\|e\|_{L^{2}(0, n \Delta t) \leq}^{2} \leq & 5 \pi L^{2}\left[\sum_{m=0}^{\prime n}\|e\|_{L^{2}(0, m \Delta t)}^{2}\right] \\
& +C \Delta t+5\left\|\sum_{j=1}^{N} \gamma_{j}\left[s^{(j)}-\bar{s}^{(j)}\right]\right\|_{L^{2}(0, M \Delta t)}^{2} .
\end{aligned}
$$

The near-orthogonality of the $s^{(j)}$ and the $\bar{s}^{(k)}$ enables a bound on the final term (use Appendix $\mathrm{C}$ with $\beta_{j}=\gamma_{j}$ ) and the required result follows from a Gronwall argument.

To verify this result numerically we performed a computer simulation for [MP1] with $f \equiv 0$ using method (2.9), (2.10). The parameters for the experiment were $\alpha=1, \theta=0$, and $N \Delta t=\frac{\pi}{10}$ for $N=2000,4000,8000$, and 16,000 . We observed that the $L^{2}(0, t)$ error converged at a rate of $\mathcal{O}\left(\Delta t^{0.4831}\right)$, an improvement over the proven bound $\mathcal{O}\left(\sqrt{\log \Delta t^{-1}} \Delta t^{1 / 3}\right)$; see Figure 3.1.

Note that the convergence rate was determined as the slope of the leastsquares fit line through the log-log data points in Figure 3.1. This methodology is employed for determining all numerical convergence rates in this paper.

We now comment on what happens to the numerical method if $\theta+\alpha \neq 1$. In this case $Z^{n}$ has an extra contribution

$$
(1-\theta-\alpha) \Delta t \sum_{j=1}^{N}\left[1-\cos \left(\phi_{j} n\right)\right] .
$$

By use of Appendix $\mathrm{C}$ with $\beta_{j}=\Delta t=\zeta / N \leq \zeta / j$ it follows that

$$
\Delta t \sum_{j=1}^{N} \cos \left(\phi_{j} n\right)=\Delta t \sum_{j=1}^{N} \cos (j n \Delta t)+\delta_{1},
$$



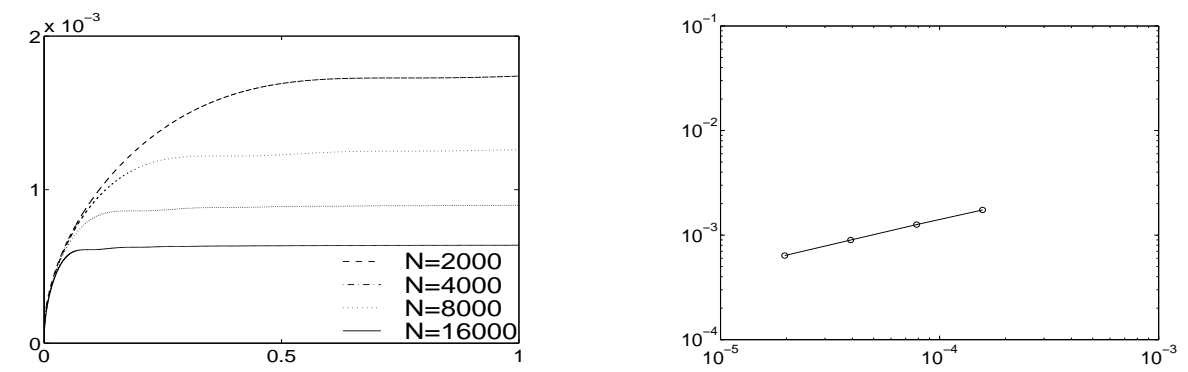

Figure 3.1: (a) $L^{2}(0, t)$ error curves from [MP1] with $f \equiv 0$ using method (2.9), (2.10) with $N \Delta t=\frac{\pi}{10}$ and $\theta=0, \alpha=1$. (b) Log-log plot for the convergence rate of $L^{2}(0,1)$ error as a function of $\Delta t$; the approximate slope is 0.4831 .

where

$$
\left\|\delta_{1}\right\|_{L^{2}(0, M \Delta t)}^{2}=\mathcal{O}\left(\log \left|\Delta t^{-1}\right| \Delta t^{2 / 3}\right) .
$$

Summing the resulting geometric series found by writing the cosine as the real part of a complex exponential (by use of [4], 1.342(2)) shows that $Z^{n}$ has an extra contribution

$$
(1-\theta-\alpha)\left\{r+\delta_{2}\right\}
$$

where

$$
\left\|\delta_{2}\right\|_{L^{2}(0, M \Delta t)}^{2}=\mathcal{O}\left(\log \left|\Delta t^{-1}\right| \Delta t^{2 / 3}\right) .
$$

From this it follows that for $\theta+\alpha \neq 1$ and under (1.1), the numerical method approximates the ODE

$$
\dot{y}=f(y), \quad y(0)=z_{0}+\frac{\pi}{2}+(1-\theta-\alpha) r
$$

instead of the true limiting equation (2.5). Thus the numerical method accurately computes the wrong limit. More precisely we have:

Theorem 3.2 Consider $\left\{Z^{n}\right\}_{n \geq 0}$ solving [MP1] numerically with $\theta+\alpha \neq 1$ and $N \Delta t=r<2$ and $\left\{y^{n}\right\}_{n \geq 0}$ the projection of $y(t)$ solving (3.4) onto the grid. Then, for $n \Delta t \in[0, \pi]$,

$$
\|y-Z\|_{L^{2}(0, n \Delta t)}^{2} \leq C(n \Delta t) \log \left|\Delta t^{-1}\right| \Delta t^{2 / 3} .
$$

This result has been verified via a computer simulation analogous to the experiment illustrated in Figure 3.1. Again we observed that the $L^{2}(0, t)$ error converged at rate $\mathcal{O}\left(\Delta t^{1 / 2}\right)$, suggesting the theoretical upper bounds from Theorem 3.1 and Theorem 3.2 may be improved.

\section{Strong Approximation of White Noise}

We employ the notation introduced in the previous section. Our main result here is encapsulated in the following theorem. 
Theorem 4.1 Consider $\left\{Z^{n}\right\}_{n \geq 1}$ solving [MP2] numerically with $N \Delta t=$ $r<2$. Then, for $n \Delta t \in[0, \pi]$,

$$
\sup _{0 \leq m \leq n-1} \mathbb{E}\left|z^{m}-Z^{m}\right|^{2} \leq C(n \Delta t) \Delta t^{2 / 3}
$$

Proof By (2.15) we can almost surely rewrite (2.16) as

$$
z(t)=z_{0}+\int_{0}^{t} f(z(s)) d s+\frac{\eta_{0} t}{\sqrt{\pi}}+\sqrt{\frac{2}{\pi}} \sum_{j=1}^{\infty} \eta_{j} \frac{\sin (j t)}{j} .
$$

Using the limited regularity of solutions $z(t)$ to the SDE (2.6) it follows that

$$
\mathbb{E}\left|\int_{0}^{t} f(z(s)) d s-\Delta t \sum_{m=0}^{n} f\left(z^{m}\right)\right|^{2} \leq C \Delta t .
$$

Hence from (3.1) we obtain, using $a_{j}=\eta_{j}$ which is distributed as $\mathcal{N}(0,1)$,

$$
\begin{aligned}
\mathbb{E}\left|e^{n}\right|^{2} \leq & 6 \pi L^{2} \sum_{m=0}^{\prime n} \mathbb{E}\left|e^{m}\right|^{2}+6 C \Delta t \\
& +3 \pi \sum_{j \geq N+1} \frac{1}{j^{2}}+3 \pi \sum_{j=1}^{N}\left(j^{-1}-\gamma_{j}\right)^{2} \\
& +6 \sum_{j=1}^{N} \gamma_{j}^{2}\left[\sin (j n \Delta t)-\sin \left(\phi_{j} n\right)\right]^{2} \\
& +24 N \Delta t^{2}(\theta+\alpha-1)^{2} .
\end{aligned}
$$

But

$$
\begin{aligned}
\sum_{j=1}^{N} \gamma_{j}^{2}\left[\sin (j n \Delta t)-\sin \left(\phi_{j} n\right)\right]^{2} & \leq \mathcal{O}\left[\sum_{j=1}^{N^{\alpha}} j^{4} \Delta t^{4}+\sum_{j \geq N^{\alpha}} \frac{1}{j^{2}}\right] \\
& =\mathcal{O}\left(\Delta t^{4} N^{5 \alpha}+N^{-\alpha}\right) .
\end{aligned}
$$

Choosing $\alpha=2 / 3$ we obtain, also using (3.3),

$$
\mathbb{E}\left|e^{n}\right|^{2} \leq 6 \pi L^{2} \sum_{m=0}^{n}{ }^{\prime} \mathbb{E}\left|e^{m}\right|^{2}+\mathcal{O}\left(\Delta t^{2 / 3}\right)
$$

and the required result follows by a Gronwall argument.

Once again we verified our result numerically, solving [MP2] with $\theta=$ $\alpha=0, f(z)=-z$, and $N \Delta t=1$ for $N=2000,4000,8000$, and 16,000. Due to the highly oscillatory behaviour of a single realization path, we depict the $L^{2}(0, t)$ error, observing an approximate convergence rate of $\mathcal{O}\left(\Delta t^{0.3997}\right)$ for this single realization; see Figure 4.1. Note that Theorem 4.1 involves an average over all paths whilst our experiment is for a single path. 

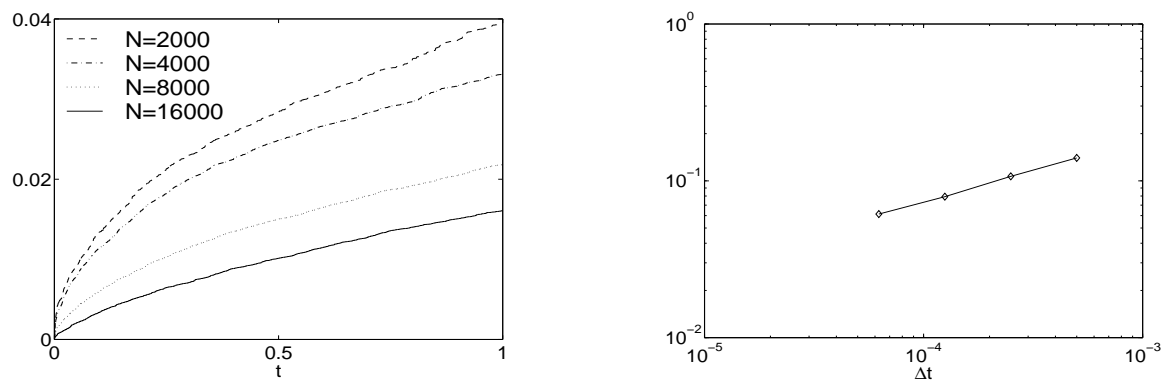

Figure 4.1: (a) $L^{2}(0, t)$ error curves from [MP2] with $f(z)=-z$ using method (2.9), (2.10) with $N \Delta t=1$ and $\theta=\alpha=0$. (b) Log-log plot for the convergence rate of $L^{2}(0,1)$ as a function of $\Delta t$; the approximate slope is 0.3997 .

\section{Weak Numerical Approximation of White Noise}

Our analysis is confined to the simple case where $f \equiv 0$ so that the desired weak convergence properties of [MP2] solved numerically should approximate those of pure diffusion. This enables us to use Fourier techniques. After the analysis some experiments will be presented to show that the result is more general than that presented in the following theorem and can be extended to non-zero $f$. To analyze the case of non-zero $f$ would require more sophisticated techniques, such as those described in [1].

Theorem 5.1 Consider $\left\{Z^{n}\right\}_{n \geq 1}$ solving [MP2] numerically with $N \Delta t=$ $r<2$. Then, for $n \Delta t \in[0, \pi]$,

$$
\sup _{z_{0} \in R}\left|\mathbb{E} g(z(n \Delta t))-\mathbb{E} g\left(Z^{n}\right)\right| \leq C \begin{cases}N^{(1-\beta) / 3}, & 1<\beta<3, \\ N^{-2 / 3} \log (1+N), & \beta=3, \\ N^{-2 / 3}, & \beta>3,\end{cases}
$$

where $C=C\left(\beta, C_{1}\right)$ with $\beta$ and $C_{1}$ as in Hypothesis $H$.

Proof We let $p_{\mathrm{N}, \Delta t}^{n}(z ; \omega)$ denote the pdf for $Z^{n}$ solving $(2.10)$, for each fixed $\omega$, and

$$
\bar{p}_{\mathrm{N}, \Delta t}^{n}(z)=\mathbb{E} p_{\mathrm{N}, \Delta t}^{n}(z ; \omega) .
$$

Since $f(\cdot) \equiv 0$ we have, by Lemma 2.4 ,

$$
Z^{n}=z_{0}+s^{n}
$$

where

$$
s^{n}=\frac{1}{\pi} \eta_{0}(n \Delta t)+\sum_{j=1}^{N} \sqrt{\frac{2}{\pi}} \eta_{j}\left\{\gamma_{j} \sin \left(\phi_{j} n\right)+2 \Delta t(1-\theta-\alpha) \sin ^{2}\left(\phi_{j} n / 2\right)\right\} .
$$

If $p_{\mathrm{N}, \Delta t}^{0}(z ; \omega)=p_{0}(z)$ then $p_{\mathrm{N}, \Delta t}^{n}(z ; \omega)=p_{0}\left(z-s^{n}\right)$; assuming that $p_{0}(z)=$ $\delta\left(z-z_{0}\right)$ we obtain

$$
\bar{p}_{\mathrm{N}, \Delta t}^{n}(z)=\frac{1}{2 \pi} \int_{-\infty}^{\infty} e^{i k\left(z_{0}-z\right)} \psi_{\mathrm{N}, \Delta t}^{n}(k) d k,
$$


where $\psi_{\mathrm{N}, \Delta t}^{n}(k)$ is the characteristic function for $s^{n}=s^{n}(\omega)$; i.e.

$$
\begin{aligned}
\psi_{\mathrm{N}, \Delta t}^{n}(k) & =\mathbb{E} \exp \left\{i k s^{n}(t ; \omega)\right\} \\
& =\exp \left\{-\frac{1}{2} k^{2}\left[\frac{(n \Delta t)^{2}}{\pi}+S_{2}^{N}\right]\right\},
\end{aligned}
$$

where

$$
S_{2}^{N}=\frac{2}{\pi} \sum_{j=1}^{N}\left[\gamma_{j} \sin \left(n \phi_{j}\right)+2 \Delta t(1-\theta-\alpha) \sin ^{2}\left(\phi_{j} n / 2\right)\right]^{2} .
$$

By (2.27) and (2.29) we deduce that

$$
\left|\psi_{\mathrm{N}}(k, n \Delta t)-\psi_{\mathrm{N}, \Delta t}^{n}(k)\right| \leq e^{-\frac{k^{2}(n \Delta t)^{2}}{2 \pi}}\left[1-e^{-k^{2}\left|S_{1}^{N}-S_{2}^{N}\right|}\right] .
$$

But

$$
\begin{aligned}
\frac{\pi}{2}\left|S_{1}^{N}-S_{2}^{N}\right|=\mid & \mid \sum_{j=1}^{N}\left[\gamma_{j}^{2}-\frac{1}{j^{2}}\right] \sin ^{2}(j n \Delta t) \\
& +\sum_{j=1}^{N} \gamma_{j}^{2}\left[\sin ^{2}\left(n \phi_{j}\right)-\sin ^{2}(j n \Delta t)\right] \\
& +\sum_{j=1}^{N} 4 \Delta t(1-\theta-\alpha) \gamma_{j} \sin \left(n \phi_{j}\right) \sin ^{2}\left(n \phi_{j} / 2\right) \\
& +\sum_{j=1}^{N} 4 \Delta t^{2}(1-\theta-\alpha)^{2} \sin ^{4}\left(n \phi_{j} / 2\right) \mid .
\end{aligned}
$$

Thus, by (3.2),

$$
\begin{aligned}
\frac{\pi}{2}\left|S_{1}^{N}-S_{2}^{N}\right| \leq & \sum_{j=1}^{N} \frac{C}{j}\left|\gamma_{j}-\frac{1}{j}\right|+\sum_{j=1}^{N} \frac{C}{j^{2}}\left|\sin \left(n \phi_{j}\right)-\sin (j n \Delta t)\right| \\
& +\sum_{j=1}^{N} \frac{C \Delta t}{j}+\sum_{j=1}^{N} C \Delta t^{2} \\
\leq & \mathcal{O}\left(\log \left|\Delta t^{-1}\right| \Delta t\right)+\sum_{j=1}^{N} \frac{C}{j^{2}}\left|\sin \left(n \phi_{j}\right)-\sin (j n \Delta t)\right| .
\end{aligned}
$$

But $\phi_{j}=j \Delta t+\mathcal{O}\left(j^{3} \Delta t^{3}\right)$ and so, by choosing $\beta=2 / 3$ and noting that $n \Delta t=\mathcal{O}(1)$,

$$
\sum_{j=1}^{N} \frac{C}{j^{2}}\left|\sin \left(n \phi_{j}\right)-\sin (j n \Delta t)\right| \leq \mathcal{O}\left(\sum_{j=1}^{N^{\beta}} j \Delta t^{2}+\sum_{j \geq N^{\beta}} \frac{1}{j^{2}}\right)=\mathcal{O}\left(\Delta t^{2 / 3}\right) .
$$

Thus

$$
\left|\psi_{\mathrm{N}}(k, n \Delta t)-\psi_{\mathrm{N}, \Delta t}^{n}(k)\right| \leq \mathrm{e}^{-\frac{k^{2}(n \Delta t)^{2}}{2 \pi}}\left[1-\mathrm{e}^{-\frac{C k^{2}}{N^{2} / 3}}\right] .
$$

Now, by Parseval's identity,

$$
\begin{gathered}
\mathbb{E} g\left(z_{\mathrm{N}}(n \Delta t)\right)-\mathbb{E} g\left(Z^{n}\right)=\int_{-\infty}^{\infty}\left[\bar{p}_{\mathrm{N}}(z, n \Delta t)-\bar{p}_{\mathrm{N}, \Delta t}^{n}(z)\right] g(z) d z \\
=\frac{1}{2 \pi} \int_{-\infty}^{\infty} \mathrm{e}^{i k\left(z_{0}-z\right)}\left[\psi_{\mathrm{N}}^{n}(k, n \Delta t)-\psi_{\mathrm{N}, \Delta t}^{n}(k)\right] \hat{g}(k) d k .
\end{gathered}
$$

Thus, by Hypothesis H,

$$
\left|\mathbb{E} g\left(z_{\mathrm{N}}(n \Delta t)\right)-\mathbb{E} g\left(Z^{n}\right)\right| \leq \frac{C_{1}}{\pi} \int_{0}^{\infty} \mathrm{e}^{-\frac{k^{2}(n \Delta t)^{2}}{2 \pi}}\left[1-\mathrm{e}^{-\frac{C k^{2}}{N^{2} / 3}}\right](1+k)^{-\beta} d k .
$$




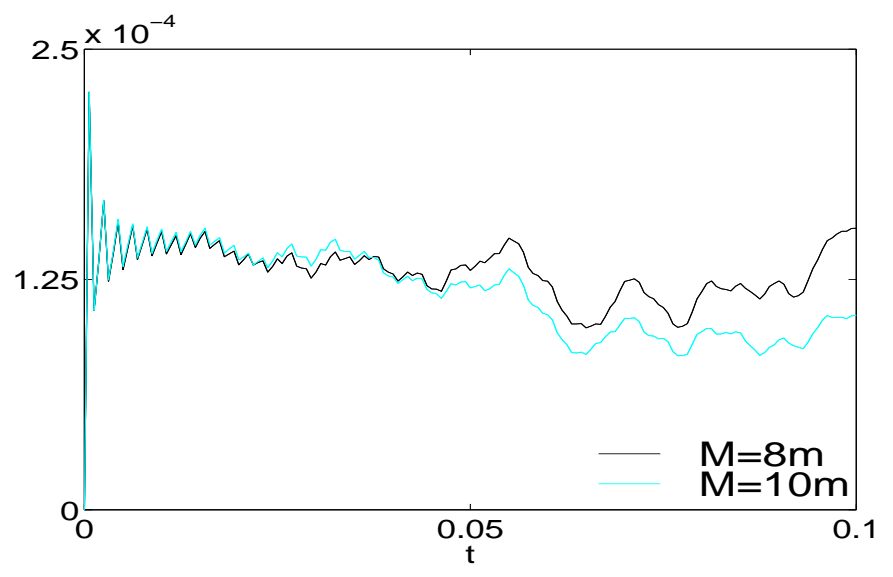

Figure 5.1: $\left|t-\mathbb{E} g\left(Z^{n}\right)\right|$ up to time $t=0.1$ for $N=1600$ and $\mathbb{E} g\left(Z^{n}\right)$ approximated with $8 \mathrm{~m}$ and $10 \mathrm{~m}$ realizations.

Analysis analogous to that at the end of the proof of Theorem 2.3 (using Appendix A) but with $N^{-1}$ replaced by $N^{-2 / 3}$ gives the required result.

For our first numerical experiment we solved [MP2] with $f \equiv 0$ and chose $g(z)=z^{2}$. Thus $z$ is simply Brownian motion and $\mathbb{E} g(z(t))=t$. However, calculating $\mathbb{E} g\left(Z^{n}\right)$ accurately is a computationally intense task since, by Theorem 5.1, for sufficiently smooth $g$,

$$
\left|\mathbb{E} g(z(n \Delta t))-\mathbb{E} g\left(Z^{n}\right)\right| \leq \mathcal{O}\left(N^{-2 / 3}\right) .
$$

Hence to determine the rate of convergence, the statistical error in estimating the expectation $\mathbb{E} g\left(Z^{n}\right)$ must be insignificant compared to this bound. Furthermore, the variance of $g\left(Z^{n}\right)$ increases as $n$ increases, thus requiring more realizations to accurately estimate this expectation.

Figure 5.1 shows the difference in numerical estimates of the expectations up to time $t=0.1$ using 8 million and 10 million realizations with $N=1600$. Note that the curves differ for $t>0.05$ in the two cases, even for $\mathcal{O}\left(10^{7}\right)$ realizations (though the relative error $\left|t-\mathbb{E} g\left(Z^{n}\right)\right| / t$ is better behaved). Moreover, for large $t(t \approx 1)$, this statistical error overwhelms the quantity of interest $\left|\mathbb{E} g(z(n \Delta t))-E g\left(Z^{n}\right)\right|$. This suggests that to examine the rate of weak convergence we are restricted to small time intervals and a large number of realizations. Note that for $t \leq 0.05$ both estimates of $\mathbb{E} g\left(Z^{n}\right)$ are fairly well converged and, in fact, deviations are negligible in comparison with the quantity $\left|t-\mathbb{E} g\left(Z^{n}\right)\right|$ which we wish to estimate.

For our experiment we examined weak convergence up to time $t=0.05$ with $\theta=0, \alpha=0$ and $N=200,400,800$, and 1600. We observed that $\mid \mathbb{E} g\left(z(n \Delta t)-\mathbb{E} g\left(Z^{n}\right) \mid\right.$ at $t=0.05$ converged at approximate rate of $\mathcal{O}\left(\Delta t^{0.9196}\right)$, an improvement over the theoretical rate of $\mathcal{O}\left(\Delta t^{2 / 3}\right)$. These results are depicted in Figure 5.2.

Finally we repeated this experiment using a non-zero forcing function: $f(z)=z-z^{3}$. We observed a convergence rate of $\mathcal{O}\left(\Delta t^{0.9313}\right)$, suggesting that the theory can be extended to incorporate non-zero $f$. 

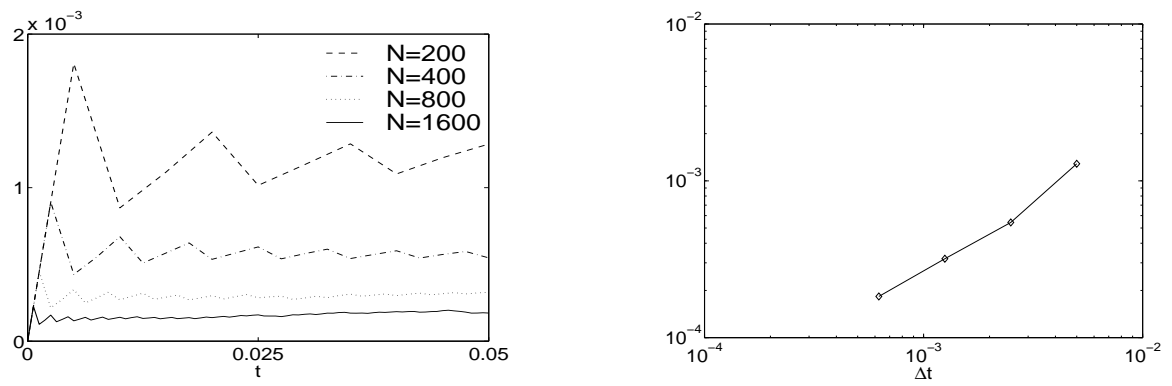

Figure 5.2: (a) $\left|\mathbb{E} g(z(n \Delta t))-\mathbb{E} g\left(Z^{n}\right)\right|$ error curves from [MP2] with $f \equiv 0$ using method (2.9), (2.10) with $N \Delta t=1$ and $\theta=\alpha=0$. (b) Log-log plot for the convergence rate of error at $t=0.05$ as a function of $\Delta t$; the approximate slope is 0.9196 .

\section{References}

[1] G. Blankenship and G.C. Papanicolaou, Stability and control of stochastic systems with wide-band noise disturbances. I. SIAM J. Appl. Math. 34(1978), 437-476.

[2] K. Dekker and J. Verwer, Stability of Runge-Kutta methods for stiff nonlinear differential equations. North-Holland, Amsterdam, 1984.

[3] G.W. Ford and M. Kac, On the quantum Langevin equation. J. Stat. Phys. 46(1987), 803-810.

[4] I.S. Gradshteyn and I.M. Ryzhik, Table of Integrals, Series and Products. Academic Press, New York, 1965.

[5] P. Kloeden and E. Platen, Numerical Solution of Stochastic Differential Equations. Springer, 1994.

[6] N.V. Krylov, Introduction to the Theory of Diffusion Processes. AMS Translations of Monographs, Volume 142.

[7] L. Petzold, L.O. Jay and J. Yen, Numerical solution of highly oscillatory ordinary differential equations. Acta Numerica 6(1997), 437-483.

[8] A.M. Stuart and J.O. Warren, Analysis and experiments for a computational model of a heat bath, Technical Report SCCM-98-17, http://wwwsccm.stanford.edu/pub/sccm/sccm98-17.ps.gz.

[9] W. Thirring, Quantum Mechanics of Large Systems. Springer-Verlag, New York, 1983.

[10] G.E. Uhlenbeck and G.W. Ford, Lectures in Statistical Mechanics. AMS, Rhode Island, 1963. 


\section{Appendix A}

Our aim in this Appendix is to prove the estimate (2.36). The starting point is the following decomposition:

$$
\begin{aligned}
\int_{0}^{\infty} & \mathrm{e}^{-\frac{k^{2} t^{2}}{2 \pi}}(1+k)^{-\beta} \min \left(1, \frac{k^{2}}{\pi N}\right) d k \\
& =\int_{0}^{\sqrt{\pi N}} \frac{\mathrm{e}^{-\frac{k^{2} t^{2}}{2 \pi}}}{(1+k)^{\beta}} \frac{k^{2}}{\pi N} d k+\int_{\sqrt{\pi N}}^{\infty} \frac{\mathrm{e}^{-\frac{k^{2} t^{2}}{2 \pi}}}{(1+k)^{\beta}} d k \equiv I+I I .
\end{aligned}
$$

We begin by estimating term $I$ for $t>0$.

$$
\begin{aligned}
I & =\frac{t^{-3}}{\pi N} \int_{0}^{\sqrt{\pi N}} \frac{\mathrm{e}^{-\frac{k^{2} t^{2}}{2 \pi}}}{\left(1+\frac{k t}{t}\right)^{\beta}}(k t)^{2} d(k t) \\
& =\frac{t^{\beta-3}}{\pi N} \int_{0}^{t \sqrt{\pi N}} \frac{\mathrm{e}^{-\frac{u^{2}}{2 \pi}}}{(t+u)^{\beta}} u^{2} d u .
\end{aligned}
$$

1a) First, suppose that $0<t \sqrt{\pi N} \leq 1$. Then, assuming $\beta \neq 3$,

$$
\begin{aligned}
I & \leq \frac{t^{\beta-3}}{\pi N} \int_{0}^{t \sqrt{\pi N}}\left(\frac{u}{t+u}\right)^{2} \frac{d u}{(t+u)^{\beta-2}} \\
& \leq\left.\frac{t^{\beta-3}}{\pi N} \frac{(t+u)^{3-\beta}}{3-\beta}\right|_{0} ^{t \sqrt{\pi N}}, \\
& =\frac{t^{\beta-3}}{\pi N} \frac{t^{3-\beta}}{3-\beta}\left[(1+\sqrt{\pi N})^{3-\beta}-1\right] \\
& =\frac{C}{N}\left[(1+\sqrt{\pi N})^{3-\beta}-1\right] \\
& \leq \begin{cases}C N^{-1} & \text { when } \beta>3, \\
C N^{(1-\beta) / 2} & \text { when } 1<\beta<3,\end{cases}
\end{aligned}
$$

where $C=C(\beta)$ is a positive constant. Similarly, when $\beta=3$,

$$
\begin{aligned}
I & \leq \frac{1}{\pi N} \int_{0}^{t \sqrt{\pi N}} \frac{d u}{t+u}=\left.\frac{1}{\pi N} \log (t+u)\right|_{0} ^{t \sqrt{\pi N}} \\
& =\frac{1}{\pi N}(\log (t+t \sqrt{\pi N})-\log t) \\
& =\frac{1}{\pi N} \log (1+\sqrt{\pi N}) \leq C N^{-1} \log (1+N)
\end{aligned}
$$

where $C$ is a positive constant. To summarise the situation, for $0<t \sqrt{\pi N} \leq$ 1 we have that

$$
I \leq \begin{cases}C N^{(1-\beta) / 2}, & 1<\beta<3 \\ C N^{-1} \log (1+N), & \beta=3 \\ C N^{-1}, & \beta>3\end{cases}
$$


where $C=C(\beta)$ is a positive constant.

1b) Now suppose that $t \sqrt{\pi N} \geq 1$. Then,

$$
\left(\frac{1}{t}\right)^{3-\beta} \leq(\sqrt{\pi N})^{3-\beta}, \quad 1<\beta<3 .
$$

We shall make use of this below. First, since $(t+u)^{-\beta} u^{2} \leq(t+u)^{2-\beta}$ for $0 \leq u \leq 1$, and $(t+u)^{-\beta} \leq 1$ for $u \geq 1$, we have from (A.2) that

$$
\begin{aligned}
I & \leq \frac{t^{\beta-3}}{\pi N}\left(\int_{0}^{1} \frac{\mathrm{e}^{-\frac{u^{2}}{2 \pi}}}{(t+u)^{\beta}} u^{2} d u+\int_{1}^{\infty} \frac{e^{-\frac{u^{2}}{2 \pi}}}{(t+u)^{\beta}} u^{2} d u\right) \\
& \leq \frac{C t^{\beta-3}}{N}\left(\int_{0}^{1} \frac{d u}{(t+u)^{\beta-2}}+1\right) \\
& = \begin{cases}\frac{C}{N}\left(\frac{\left(1+\frac{1}{t}\right)^{3-\beta}-1}{3-\beta}+1\right), & \beta \neq 3, \\
\frac{C}{N}\left(\log \left(1+\frac{1}{t}\right)+1\right), & \beta=3 .\end{cases}
\end{aligned}
$$

Finally, using (A.4) this implies that, for $t \sqrt{\pi N} \geq 1$,

$$
I \leq \begin{cases}C N^{(1-\beta) / 2}, & 1<\beta<3, \\ C N^{-1} \log (1+N), & \beta=3, \\ C N^{-1}, & \beta>3 .\end{cases}
$$

From (A.3) and (A.5) we deduce that

$$
I \leq \begin{cases}C N^{(1-\beta) / 2}, & 1<\beta<3, \\ C N^{-1} \log (1+N), & \beta=3, \\ C N^{-1}, & \beta>3 .\end{cases}
$$

for all $t>0$, where $C=C(\beta)$ is a positive constant.

Now we consider term $I I$ in (A.1).

$$
\begin{aligned}
I I & =\int_{\sqrt{\pi N}}^{\infty} \frac{\mathrm{e}^{-\frac{k^{2} t^{2}}{2 \pi}}}{(1+k)^{\beta}} d k \\
& =\frac{1}{t} \int_{\sqrt{\pi N}}^{\infty} \frac{\mathrm{e}^{-\frac{(k t)^{2}}{2 \pi}}}{\left(1+\frac{k t}{t}\right)^{\beta}} d(k t)=t^{\beta-1} \int_{t \sqrt{\pi N}}^{\infty} \frac{\mathrm{e}^{-\frac{u^{2}}{2 \pi}}}{(t+u)^{\beta}} d u .
\end{aligned}
$$

2a) Suppose that $0<t \sqrt{\pi N} \leq 1$.

$$
\begin{aligned}
I I & \leq t^{\beta-1}\left\{\int_{t \sqrt{\pi N}}^{1} \frac{\mathrm{e}^{-\frac{u^{2}}{2 \pi}}}{(t+u)^{\beta}} d u+\int_{1}^{\infty} \frac{\mathrm{e}^{-\frac{u^{2}}{2 \pi}}}{(t+u)^{\beta}} d u\right\} \\
& \leq t^{\beta-1}\left\{\int_{t \sqrt{\pi N}}^{1} \frac{1}{(t+u)^{\beta}} d u+\int_{1}^{\infty} \mathrm{e}^{-\frac{u^{2}}{2 \pi}} d u\right\} \\
& \leq C t^{\beta-1}\left\{\left.\frac{(t+u)^{1-\beta}}{1-\beta}\right|_{t \sqrt{\pi N}} ^{1}+1\right\} \\
& =C t^{\beta-1}\left\{\frac{(1+t)^{1-\beta}-t^{1-\beta}(1+\sqrt{\pi N})^{1-\beta}}{1-\beta}+1\right\} .
\end{aligned}
$$


Thus,

$$
\begin{aligned}
I I & \leq C\left(\left|t^{\beta-1}(1+t)^{1-\beta}-(1+\sqrt{\pi N})^{1-\beta}\right|+t^{\beta-1}\right) \\
& \leq C\left(N^{(1-\beta) / 2}+t^{\beta-1}\right) \\
& \leq C N^{(1-\beta) / 2}
\end{aligned}
$$

where in the transition to the last line we made use of the fact that $t \sqrt{\pi N} \leq 1$. Here $C=C(\beta)$ is a positive constant.

2b) Now suppose that $t \sqrt{\pi N} \geq 1$. Then,

$$
\begin{aligned}
I I & \leq t^{\beta-1} \int_{t \sqrt{\pi N}}^{\infty} \frac{\mathrm{e}^{-\frac{u^{2}}{2 \pi}}}{(t+u)^{\beta}} d u \\
& \leq t^{\beta-1} e^{-\frac{t^{2} N}{2}} \int_{t \sqrt{\pi N}}^{\infty} \frac{d u}{(t+u)^{\beta}} \\
& =t^{\beta-1} e^{-\frac{t^{2} N}{2}} \frac{(t+t \sqrt{\pi N})^{1-\beta}}{\beta-1} \\
& =e^{-\frac{t^{2} N}{2}} \frac{(1+\sqrt{\pi N})^{1-\beta}}{\beta-1} \\
& \leq \frac{\mathrm{e}^{-\frac{1}{2 \pi}}}{\beta-1} N^{(1-\beta) / 2}\left(\sqrt{\pi}+\frac{1}{\sqrt{N}}\right)^{1-\beta} \\
& \leq C N^{(1-\beta) / 2}
\end{aligned}
$$

where $C=C(\beta)$ is a positive constant. Thus, to summarise,

$$
I I \leq C N^{(1-\beta) / 2}, \quad \beta>1,
$$

with $C=C(\beta)$ a positive constant. Finally, substituting the bounds (A.6) and (A.7) into (A.1) we arrive at the estimate (2.36).

\section{Appendix $B$}

Proof of Proposition 2.5: We begin the proof by observing that

$$
\begin{aligned}
\left|\bar{p}_{\mathrm{N}}(z, t)-p(z, t)\right| & =\frac{1}{2 \pi}\left|\int_{-\infty}^{\infty} \mathrm{e}^{i k\left(z_{0}-z\right)}\left[\mathrm{e}^{-\frac{1}{2} k^{2} t}-\psi_{N}(k, t)\right] d k\right| \\
& \leq \int_{-\infty}^{\infty}\left|e^{-\frac{1}{2} k^{2} t}-\psi_{N}(k, t)\right| d k .
\end{aligned}
$$


We substitute (2.31) into (B.1) to conclude that

$$
\begin{aligned}
\left|\bar{p}_{\mathrm{N}}(z, t)-p(z, t)\right| \leq \int_{-\infty}^{\infty} \mathrm{e}^{-\frac{k^{2} t^{2}}{2 \pi}}\left[1-\mathrm{e}^{-\frac{k^{2}}{\pi N}}\right] d k \\
=\frac{1}{t} \int_{-\infty}^{\infty} \exp \left\{-\frac{(k t)^{2}}{2 \pi}\right\} d(k t) \\
\quad-\frac{1}{\sqrt{t^{2}+\frac{2}{N}}} \int_{-\infty}^{\infty} \exp \left\{-\frac{\left(k \sqrt{t^{2}+\frac{2}{N}}\right)^{2}}{2 \pi}\right\} d\left(k \sqrt{t^{2}+\frac{2}{N}}\right) \\
=C_{0}\left[\frac{1}{t}-\frac{1}{\sqrt{t^{2}+\frac{2}{N}}}\right],
\end{aligned}
$$

where we put

$$
C_{0}=\int_{-\infty}^{\infty} \exp \left\{-\frac{s^{2}}{2 \pi}\right\} d s
$$

Next, we note the elementary inequality

$$
1-\frac{1}{(1+2 y)^{1 / 2}} \leq \min (1, y), \quad y \geq 0
$$

Choosing $y=1 /\left(N t^{2}\right)$ in (B.3) we deduce that

$$
1-\frac{1}{\left(1+\frac{2}{N t^{2}}\right)^{1 / 2}} \leq \min \left(1, \frac{1}{N t^{2}}\right) .
$$

Consequently,

$$
\left|\bar{p}_{N}(z, t)-p(z, t)\right| \leq \frac{C_{0}}{t} \min \left(1, \frac{1}{N t^{2}}\right)
$$

After multiplying (B.4) by $t^{\alpha}, \alpha>0$, and integrating with respect to $t$ between 0 and $T$, we obtain

$$
\begin{aligned}
& \int_{0}^{T} t^{\alpha}\left|\bar{p}_{N}(z, t)-p(z, t)\right| d t \\
& \quad=\int_{0}^{1 / \sqrt{N}} t^{\alpha}\left|\bar{p}_{N}(z, t)-p(z, t)\right| d t+\int_{1 / \sqrt{N}}^{T} t^{\alpha}\left|\bar{p}_{N}(z, t)-p(z, t)\right| d t \\
& \quad \leq C_{0} \int_{0}^{1 / \sqrt{N}} t^{\alpha-1} d t+\frac{C_{0}}{N} \int_{1 / \sqrt{N}}^{T} t^{\alpha-3} d t \equiv I+I I
\end{aligned}
$$

Elementary calculations show that

$$
I=\frac{1}{\alpha} N^{-\alpha / 2}, \quad \alpha>0
$$


and

$$
I I=C \begin{cases}\frac{1}{N} \frac{1}{\alpha-2}\left(1-\left(\frac{1}{\sqrt{N}}\right)^{\alpha-2}\right) & \text { for } \alpha \neq 2 \\ \frac{1}{2 N} \log N & \text { for } \alpha=2\end{cases}
$$

It follows from (B.7) that

$$
I I \leq \begin{cases}C N^{-\alpha / 2} & \text { for } 0<\alpha<2 \\ C N^{-1} \log (1+N) & \text { for } \alpha=2 \\ C N^{-1} & \text { for } \alpha>2\end{cases}
$$

where $C=C(\alpha, T)$ is a positive constant. Finally, inserting (B.6) and (B.8) into (B.5), we obtain (2.37).

Next we derive a similar bound where instead of the $L_{\infty}$ norm we have the $L^{2}$ norm under the integral sign. By Parseval's identity and (2.31)

$$
\begin{aligned}
& \left\|\bar{p}_{\mathrm{N}}(\cdot, t)-p(\cdot, t)\right\|_{L^{2}(R)}^{2} \leq C \int_{-\infty}^{\infty}\left[\mathrm{e}^{-\frac{1}{2} k^{2} t}-\psi_{N}(k, t)\right]^{2} d k \\
& \leq C \int_{-\infty}^{\infty} \mathrm{e}^{-\frac{1}{\pi} k^{2} t^{2}}\left[1-2 \mathrm{e}^{-\frac{k^{2}}{\pi N}}+\mathrm{e}^{-\frac{2 k^{2}}{\pi N}}\right] d k \\
& \leq C\left[\int_{-\infty}^{\infty} \mathrm{e}^{-\frac{k^{2} t^{2}}{\pi}} d k-2 \int_{-\infty}^{\infty} \mathrm{e}^{-\frac{k^{2}}{\pi}\left(t^{2}+N^{-1}\right)} d k+\int_{-\infty}^{\infty} \mathrm{e}^{-\frac{k^{2}}{\pi}\left(t^{2}+2 N^{-1}\right)} d k\right] \\
& \leq C\left[\frac{1}{t}-\frac{2}{\left(t^{2}+\frac{1}{N}\right)^{1 / 2}}+\frac{1}{\left(t^{2}+\frac{2}{N}\right)^{1 / 2}}\right]
\end{aligned}
$$

Now,

$$
1-2(1+y)^{-1 / 2}+(1+2 y)^{-1 / 2} \leq C_{2} \min \left(1, y^{2}\right),
$$

where $C_{2}$ is a positive constant. Taking

$$
y=\frac{1}{N t^{2}}
$$

in this inequality, we deduce that

$$
\left\|\bar{p}_{\mathrm{N}}(\cdot, t)-p(\cdot, t)\right\|_{L^{2}(R)} \leq \frac{C}{\sqrt{t}} \min \left(1, \frac{1}{N t^{2}}\right)
$$

where $C$ is a positive constant. Thus

$$
\int_{0}^{1} t^{\alpha}\left\|\bar{p}_{\mathrm{N}}(\cdot, t)-p(\cdot, t)\right\|_{L^{2}(R)} d t \leq C\left[\int_{0}^{\frac{1}{\sqrt{N}}} t^{\alpha-\frac{1}{2}} d t+\int_{\frac{1}{\sqrt{N}}}^{1} \frac{t^{\alpha-\frac{5}{2}}}{N} d t\right] .
$$

Consequently we obtain (2.38). 


\section{Appendix $\mathrm{C}$}

We wish to evaluate

$$
S:=\left\|\sum_{j=1}^{N} \beta_{j}\left[v^{(j)}-w^{(j)}\right]\right\|^{2}
$$

where

$$
\left(\begin{array}{c}
v_{n}^{(j)} \\
w_{n}^{(j)}
\end{array}\right)=\left(\begin{array}{c}
\sin \left(\phi_{j} n\right) \\
\sin (j n \Delta t)
\end{array}\right) \quad \text { or } \quad\left(\begin{array}{c}
\cos \left(\phi_{j} n\right) \\
\cos (j n \Delta t)
\end{array}\right)
$$

In the two applications of this result

$$
\left|\beta_{j}\right| \leq \frac{C}{j}
$$

Now

$$
S=\sum_{j, k=1}^{N} \beta_{j} \beta_{k}\left[\left\langle v^{(j)}, v^{(k)}\right\rangle_{M}-2\left\langle v^{(j)}, w^{(k)}\right\rangle_{M}+\left\langle w^{(j)}, w^{(k)}\right\rangle_{M}\right]
$$

where $M \Delta t=\pi$. But

$$
\begin{aligned}
\left\langle v^{(j)}, w^{(k)}\right\rangle_{M} & =\Delta t \sum_{n=0}^{M-1} v_{n}^{(j)} v_{n}^{(k)} \\
& =\frac{\Delta t}{2} \sum_{n=0}^{M-1} \cos \left[\left(\phi_{j}-\phi_{k}\right) n\right] \pm \frac{\Delta t}{2} \sum_{n=0}^{M-1} \cos \left[\left(\phi_{j}+\phi_{k}\right) n\right]
\end{aligned}
$$

with + for the cosine case and - for the sine case. Using [4], 1.342(2) it follows that

$$
\sum_{n=0}^{M-1} \cos (n x)=\frac{\sin [M x]}{2 \tan [x / 2]}+\sin ^{2}[M x / 2] .
$$

Summing two terms of this form with $x=x^{ \pm}$and

$$
x^{ \pm}=\phi_{j} \pm \phi_{k}, x^{ \pm}=\phi_{j} \pm k \Delta t, x^{ \pm}=(j \pm k) \Delta t
$$

gives the three inner products required to compute $S$.

In all three cases there is $k^{*}=k^{*}(j)$ which minimizes $x^{-}$. Since $\phi_{j}=$ $j \Delta t+\mathcal{O}\left(j^{3} \Delta t^{3}\right)$ we have $\kappa>0$ such that

$$
k^{*}(j)=j \quad \forall j \leq \kappa N^{2 / 3} .
$$

Then

$$
\left\langle v^{(j)}, v^{\left(k^{*}\right)}\right\rangle_{M}=\frac{\pi}{2}+\mathcal{O}\left(j^{2} \Delta t^{2}\right)+\mathcal{O}(\Delta t) \quad \forall j \leq \kappa N^{2 / 3}
$$

Otherwise

$$
\left|\left\langle v^{(j)}, v^{\left(k^{*}\right)}\right\rangle_{M}\right| \leq C .
$$


Using $|\tan (y)| \geq|y| / C$ for $|y| \leq y_{\max }<\pi$ we deduce that, for $k \neq k^{*}(j)$,

$$
\left|\left\langle v^{(j)}, v^{(k)}\right\rangle_{M}\right| \leq C\left[\Delta t+\Delta t \frac{\min \left\{1,\left(j^{3}+k^{3}\right) \Delta t^{2}\right\}}{\left|x^{ \pm}\right|}\right]
$$

noting that $x^{ \pm}$depends upon $j$ and $k$. From the properties of $\phi_{j}$ it follows that

$$
\sum_{k=1, k \neq k^{*}(j)}^{N} \frac{\Delta t}{\left|x^{ \pm}\right|} \leq C \log (N) .
$$

Thus if $\left|\beta_{j}\right| \leq C / j$ then, by $(D .1)-(D .3)$,

$$
\begin{aligned}
S & \leq \sum_{j=1}^{\kappa N^{2 / 3}} \frac{C}{j^{2}}\left[j^{2} \Delta t^{2}+\Delta t\right] \\
& +\sum_{j>\kappa N^{2 / 3}}^{N} \frac{C}{j^{2}} \\
& +\sum_{j=1}^{N}\left[\frac{C}{j} \min \left\{1, j^{3} \Delta t^{2}\right\} \sum_{k=1, k \neq k^{*}}^{N} \frac{\Delta t}{k\left|x^{\mp}\right|}\right] .
\end{aligned}
$$

Hence by $(D .4)$ we obtain

$$
S \leq C \log \left|\Delta t^{-1}\right| \Delta t^{2 / 3} .
$$

The required norm is found by taking the square-root of $S$ and so the result follows. 